\title{
Entropy in Multiple Equilibria, Systems with Two Different Sites ${ }^{\dagger}$
}

\author{
Gion Calzaferri \\ Department of Chemistry and Biochemistry, Freiestrasse 3, 3012 Bern, Switzerland; \\ gion.calzaferri@dcb.unibe.ch \\ + Presented at the 4th International Electronic Conference on Entropy and Its Applications, \\ 21 November-1 December 2017; Available online: http://sciforum.net/conference/ecea-4. \\ Published: 20 November 2017
}

\begin{abstract}
The influence of entropy in multiple chemical equilibria is investigated for systems with two different types of sites for Langmuir's condition, which means that the binding enthalpy of the species is the same for each type of sites and independent of those that are already bonded and that this holds for both types of sites independently. The analysis makes use of the particle distribution theory which holds for each type of sites separately. We provide physical insight by discussing an $X_{m}\{A B\} X_{n}$ system with $m=0,1, \ldots, M$ and $n=0,1, \ldots, N$ in detail. The procedure and results are exemplified for an $X_{m}\{A B\} X_{n}$ system with $M=3$ and $N=2$. A satisfactory consequence of the results is that the eleven equilibrium constants needed to describe such a system can be expressed as a function of two constants only. This is generally valid for any $X_{m}\{A B\} X_{n}$ system where the $[(M+1)(N+1)-1]$ equilibrium constants can be expressed as a function of 2 constants only. This has also implication for quantum-theoretical studies in the sense that it is sufficient to model only two reactions instead of many in order to describe the system. We have observed that it is sufficient to have two different sites in a multiple equilibrium in order to observe a characteristic of isotherms that cannot be described by Langmuir's equation. This is a result that may be useful for explaining experimental data which otherwise have not been explained satisfactory so far. Instead of inventing adsorption models it might often make sense of describing the system in terms of multiple equilibria.
\end{abstract}

Keywords: Chemical equilibria, entropy, particle distribution, Langmuir, equilibrium constants stoichiometrie-matrix

\section{Introduction}

We explained the influence of entropy in multiple chemical equilibria by studying the particle distribution for the conditions that the binding enthalpy of the species is the same for all sites and that it is independent of those that are already bonded [1]. Consequences were discussed for the insertion of guests into the one dimensional channels of a host, for dicarboxylic acids, and for cation exchange of zeolites. The validity of the results is independent of the nature and the strength of the binding. The quantitative link between the description of multiple equilibria and Langmuir's isotherm [2-4] was found to provide new insight. Multiple equilibria of objects with several equivalent binding, docking, coupling, or adsorption sites for neutral or charged species play an important role in all fields of chemistry [5-32]. We now investigate systems with two different types of sites, which we name $X_{m}\{A B\} X_{n}$, for the condition that the binding enthalpy of the species is the same for each type of sites and independent of those that are already bonded and that this holds for both types of sites independently. The analysis makes use of the particle distribution theory as described in ref. [1], which holds for each type of sites separately. The condition that the binding enthalpy of the species is the same for all sites and that it is independent of those that are already 
bonded is equivalent to the condition I. Langmuir used one hundred years ago to derive the Langmuir isotherm [2,3]. We therefore name it Langmuir's condition.

\section{Results and Discussion}

The number of distinguishable chemical objects of an $X_{m}\{A B\} X_{n}(m=0,1, \ldots, M$ and $n=0,1, \ldots$, $N)$ system is equal to $(M+1)(N+1)$. From this follows that the number of equilibria with $X$ is $[(M+$ $1)(N+1)-1$ ] which is also the number of equilibrium constants. We show that Langmuir's condition in connection with the particle distribution function allows to express the $(M+1)(N+1)-1$ equilibrium constants as a function of two different constants only. This is a simplification which allows studying systems quantitatively by experimental and theoretical means which otherwise might be difficult to handle. A numerical analysis of experimental data for a system with 5 different types of sites has been carried out based on this reasoning and has allowed to correct earlier reports on the reaction entropy of silver zeolite A [16]. We improve the physical insight by discussing a simple $X_{m}\{A B\} X_{n}$ system in detail. The notation $X_{m}\{A B\} X_{n}$ represents individual particles, a grid consisting of many sites, microporous objects, or other chemical systems. The procedure and results are exemplified for $m=0,1,2,3$ and $n=0,1,2$. The 11 equilibria and the corresponding equilibrium constants $K_{i}$ are collected in Table 1.

Table 1. Equilibria of an $X_{m}\{A B\} X_{n}$ system with $m=0,1,2,3$ and $n=0,1,2$.

\begin{tabular}{cc}
\hline$X_{2}\{A B\} X_{2}+X \rightleftharpoons X_{3}\{A B\} X_{2}$ & $K_{3}$ \\
$X\{A B\} X_{2}+X \rightleftharpoons X_{2}\{A B\} X_{2}$ & $K_{2}$ \\
$\{A B\} X_{2}+X \rightleftharpoons X\{A B\} X_{2}$ & $K_{1}$ \\
$X_{2}\{A B\} X+X \rightleftharpoons X_{3}\{A B\} X$ & $K_{6}$ \\
$X\{A B\} X+X \rightleftharpoons X_{2}\{A B\} X$ & $K_{5}$ \\
$\{A B\} X+X \rightleftharpoons X\{A B\} X$ & $K_{4}$ \\
$X_{2}\{A B\}+X \rightleftharpoons X_{3}\{A B\}$ & $K_{9}$ \\
$X\{A B\}+X \rightleftharpoons X_{2}\{A B\}$ & $K_{8}$ \\
$\{A B\}+X \rightleftharpoons X\{A B\}$ & $K_{7}$ \\
$\{A B\} X+X \rightleftharpoons\{A B\} X_{2}$ & $K_{11}$ \\
$\{A B\}+X \rightleftharpoons\{A B\} X$ & $K_{10}$ \\
\hline
\end{tabular}

We apply the stoichiometrie-matrix expression for evaluating these equlilibria $[9,10]$. Details of this procedure are reported in the appendix. The result is given in Table 2 . It is convenient to use the following notations to write the concentrations of the individual objects, namely $C_{i}$ and also $\left[X_{m}\{A B\} X_{n}\right]$, but only $[X]$ for the concentration of $X$.

Table 2. Concentrations $C_{i}$, calculated based on the equilibria in Table 1 and Equation (1); see appendix.

$\begin{array}{lll}\mathrm{C}_{1}=\left[X_{3}\{A B\} X_{2}\right] & =[\{A B\}][X]^{5} K_{1} K_{2} K_{3} K_{10} K_{11} \\ \mathrm{C}_{2}=\left[X_{2}\{A B\} X_{2}\right] & =[\{A B\}][X]^{4} K_{1} K_{2} K_{10} K_{11} \\ \mathrm{C}_{3}=\left[X\{A B\} X_{2}\right] & =[\{A B\}][X]^{3} K_{1} K_{10} K_{11} \\ \mathrm{C}_{4}=\left[X_{3}\{A B\} X\right] & =[\{A B\}][X]^{4} K_{4} K_{5} K_{6} K_{10} \\ \mathrm{C}_{5}=\left[X_{2}\{A B\} X\right] & =[\{A B\}][X]^{3} K_{4} K_{5} K_{10} \\ \mathrm{C}_{6}=[X\{A B\} X] & =[\{A B\}][X]^{2} K_{4} K_{10} \\ \mathrm{C}_{7}=\left[X_{3}\{A B\}\right] & =[\{A B\}][X]^{3} K_{7} K_{8} K_{9} \\ \mathrm{C}_{8}=\left[X_{2}\{A B\}\right] & =[\{A B\}][X]^{2} K_{7} K_{8} \\ \mathrm{C}_{9}=[X\{A B\}] & =[\{A B\}][X] K_{7} \\ \mathrm{C}_{10}=\left[\{A B\} X_{2}\right] & =[\{A B\}][X]^{2} K_{10} K_{11} \\ \mathrm{C}_{11}=[\{A B\} X] & =[\{A B\}][X] K_{10} \\ \mathrm{C}_{12}=\quad[\{A B\}] & =A_{0}-\sum_{i=1}^{11} C_{i} \\ \end{array}$


We have 11 equation available for expressing the 13 concentrations: $C_{i}, i=1,2, \ldots, 12$ and $[X]$. An additional equation is available from the fact that in a closed system the total concentration of the $X_{m}\{A B\} X_{n}$ species, which we name $A_{0}$, is constant, as expressed in Equation (1). The concentration $C_{12}$ $=[\{A B\}]$ can, hence, be determined using Equation (1). The concentration $[X]$ of the ligand $X$ that can bind to the $\{A B\}$ is the free variable.

$$
A_{0}-\sum_{i=1}^{12} C_{i}=0
$$

We need to know 11 equilibrium constants in order to describe the evolution of the concentrations $C_{i}$ of the twelve species as a function of the variable [X]. This is a difficult situation and may in many cases have as a consequence that a system cannot be handled in a satisfactory way. A very important simplification arises if Langmuir's condition applies. This may often be the case sufficiently well. Langmuir's condition implies in our example that $K_{1}, K_{4}$, and $K_{7}$ are equal. The same holds for $K_{2}, K_{5}$, and $K_{8}$ and also for $K_{3}, K_{6}$, and $K_{9}$. From this follows a further simplification from the application of the particle distribution function $f(n, r)[1,16,30]$, where $n$ is the total number of equilibria of a set and $r$ counts the individual equilibria in a set; $r=0,1, \ldots, n-1$ :

$$
f(n, r)=\frac{r}{r+1} \frac{n-r}{n-r+1}
$$

The particle distribution function describes the entropy decrease in the corresponding reaction sets, as we have discussed in detail [1]. Applying Langmuirs's condition and the particle distribution function we find the results reported in Table 3.

The very satisfactory consequence of the result shown in Table 3 is, that the eleven equilibrium constants can be expressed as a function of two constants only, namely $K_{1}$ and $K_{10}$. Inserting this in the equation shown in Table 2 we find the Equations (3) and (4), where $C_{12}(X)$ is the concentration of $\{A B\}$ expressed as a function of the concentration of $X$. This is a nice and very useful result. It allows to study the concentration of the twelve species $C_{i}, i=1,2, \ldots, 12$ as a function of the concentration $X$ by considering only 2 parameters, namely $K_{1}$ and $K_{10}$, instead of eleven. This has also implication for quantum-theoretical studies in the sense that it is sufficient to model only two reactions instead of eleven, in order to describe the system.

$$
\begin{gathered}
{\left[\begin{array}{l}
C_{1} \\
C_{2} \\
C_{3} \\
C_{4} \\
C_{5} \\
C_{6} \\
C_{7} \\
C_{8} \\
C_{9} \\
C_{10} \\
C_{11}
\end{array}\right](X)=\left[\begin{array}{c}
{\left[X_{3}\{A B\} X_{2}\right]} \\
{\left[X_{2}\{A B\} X_{2}\right]} \\
{\left[X\{A B\} X_{2}\right]} \\
{\left[X_{3}\{A B\} X\right]} \\
{\left[X_{2}\{A B\} X\right]} \\
{[X\{A B\} X]} \\
{\left[X_{3}\{A B\}\right]} \\
{\left[X_{2}\{A B\}\right]} \\
{[X\{A B\}]} \\
{\left[\{A B\} X_{2}\right]} \\
{[\{A B\} X]}
\end{array}\right]=\left[\begin{array}{c}
\left.\frac{1}{106}[X]^{5} \mathrm{~K}_{1}{ }^{3} \mathrm{~K}_{10}{ }^{2}\right] \\
\frac{1}{12}[X]^{4} \mathrm{~K}_{1}{ }^{2} \mathrm{~K}_{10}{ }^{2} \\
\frac{1}{4}[X]^{3} \mathrm{~K}_{1} \mathrm{~K}_{10}{ }^{2} \\
\frac{1}{27}[X]^{4} \mathrm{~K}_{1}{ }^{3} \mathrm{~K}_{10} \\
\frac{1}{3}[X]^{3} \mathrm{~K}_{1}{ }^{2} \mathrm{~K}_{10} \\
{[X]^{2} \mathrm{~K}_{1} \mathrm{~K}_{10}} \\
\frac{1}{27}[X]^{3} \mathrm{~K}_{1}{ }^{3} \\
\frac{1}{3}[X]^{2} \mathrm{~K}_{1}{ }^{2} \\
{[X] \mathrm{K}_{1}} \\
\frac{1}{4}[X]^{2} \mathrm{~K}_{10}{ }^{2} \\
{[X] \mathrm{K}_{10}}
\end{array}\right] C_{12}(X)} \\
11 \\
C_{12}(X)=A_{0}-\sum_{i=1}^{1} C_{i}(X)
\end{gathered}
$$


Table 3. Relation between the equilibrium constants defined in Table 1 as a consequence of Langmuirs's condition and the particle distribution function.

\begin{tabular}{ccccccccccc}
\hline$K_{1}$ & $K_{2}$ & $K_{3}$ & $K_{4}$ & $K_{5}$ & $K_{6}$ & $K_{7}$ & $K_{8}$ & $K_{9}$ & $K_{10}$ & $K_{11}$ \\
\hline & $K_{5}$ & $K_{6}$ & $K_{1}$ & $K_{2}$ & $K_{3}$ & $K_{1}$ & $K_{2}$ & $K_{3}$ & & \\
& $K_{8}$ & $K_{9}$ & & & & & & & & \\
& $f(3,2) K_{1}$ & $f(3,1) f(3,2) K_{1}$ & & & & & & & & $f(2,1) K_{10}$ \\
& $\frac{1}{3} K_{1}$ & $\frac{1}{9} K_{1}$ & $K_{1}$ & $\frac{1}{3} K_{1}$ & $\frac{1}{9} K_{1}$ & $K_{1}$ & $\frac{1}{3} K_{1}$ & $\frac{1}{9} K_{1}$ & & $\frac{1}{4} K_{10}$ \\
\hline
\end{tabular}

We illustrate the meaning of this result by calculation the concentrations $C_{i}$ for two sets of equilibrium constants $K_{1}$ and $K_{10}$, namely 50 and 1 , and 1 and 50, as a function of the concentration of $X$. A comparison of the results shown in Figures 1 and 2 with those reported in Figures 4, 5, and 7 in ref. [1] illustrates well that the behavior of the system with two types of sites is very different from that of a systems with only one type.
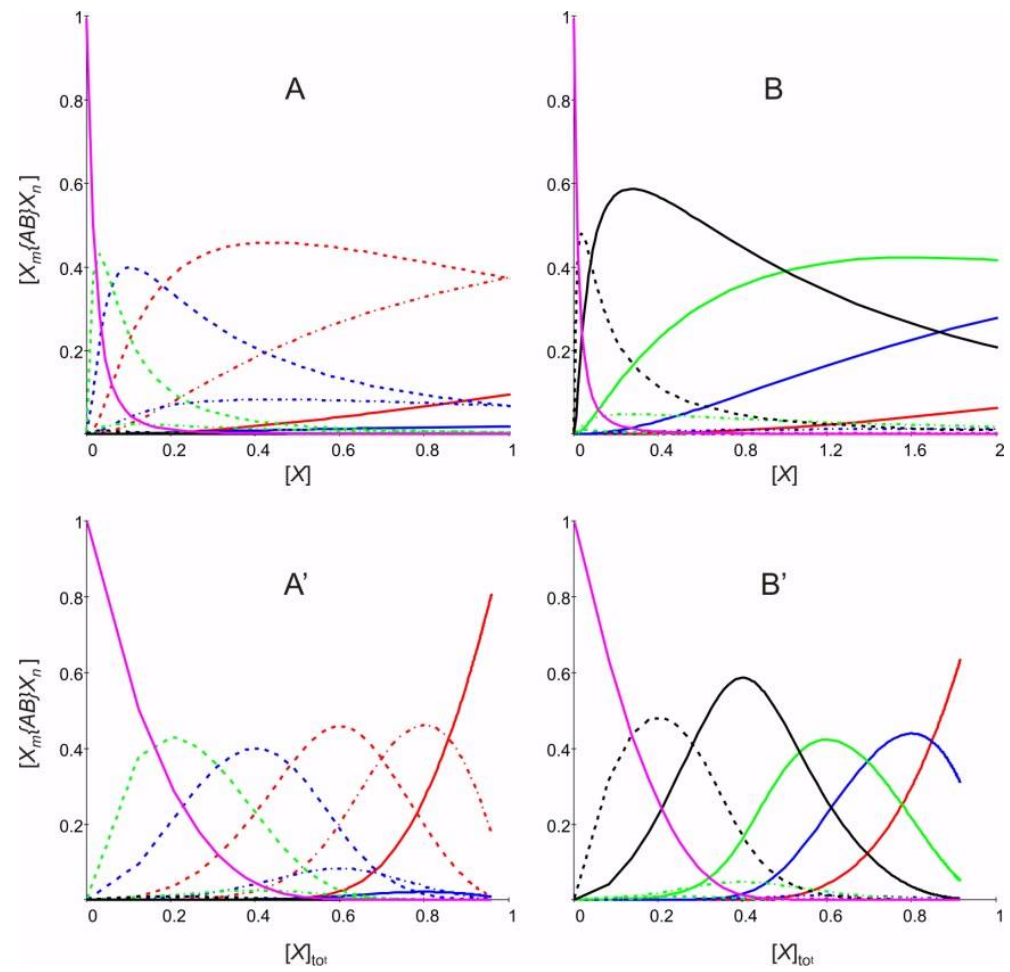

Figure 1. Concentration of $X_{m}\{A B\} X_{n}$ as a function of the concentration of $X,(\mathbf{A}, \mathbf{B})$, and versus the total concentration $[X]_{\text {tot }}$ of $X$ in the system, $\left(\mathbf{A}^{\prime}, \mathbf{B}^{\prime}\right) .\left(\mathbf{A}, \mathbf{A}^{\prime}\right)$ calculated for $K_{1}=50.0$ and $K_{10}=1.0 ;\left(\mathbf{B}, \mathbf{B}^{\prime}\right)$ calculated for $K_{1}=1.0$ and $K_{10}=50.0$. Red lines: [X $\left.\{A B\} X_{n}\right]$ (solid: $n=2$, dash: $n=1$, dash dot: $n=0$ ). Blue lines: $\left[X_{2}\{A B\} X_{n}\right]$ (solid: $n=2$, dash: $n=1$, dash dot: $\left.n=0\right)$. Green lines: [X\{AB\}Xn] (solid: $n=2$, dash: $n=1$, dash dot: $n=0)$. Black lines: $\left[\{A B\} X_{2}\right]$ solid: $[\{A B\} X]$ dash. Pink line: $[\{A B\}]=C_{12}$ (solid).

We see e.g., in Figure 1A, that the $X\{A B\} X_{n}$ appear only at the beginning for small values of $[X]$ and even more, that only $X\{A B\} X$ shows temporally a value of larger than 0.05 , while $X\{A B\} X_{2}$ always stays very small. We note that $\{A B\}$ vanishes soon and that the $X_{3}\{A B\} X_{n}$ become dominant. $\left[\{A B\} X_{2}\right]$ and $[\{A B\} X]$ always remain small. The situation changes very much in Figure $1 \mathrm{~B}$. The symmetry of the plot of the concentrations $C_{i}$ versus the total concentration $[X]_{\text {tot }}$ we have observed in Figure $4 \mathrm{~B}$ of ref. [1] has completely disappeared, however, in both cases as seen in Figure $1 \mathrm{~A}^{\prime}, \mathrm{B}^{\prime}$. We also observe that out of the 12 species $X_{m}\{A B\} X_{n}$ only few manage to evolve significant concentrations. An example with different values of $K_{1}$ and $K_{10}$ is reported in the appendix.

The fractional coverage expressed as a function of the concentration $[X]$ is of special interest, also because it can often be determined experimentally relatively easy. We show this in Figure 2. 

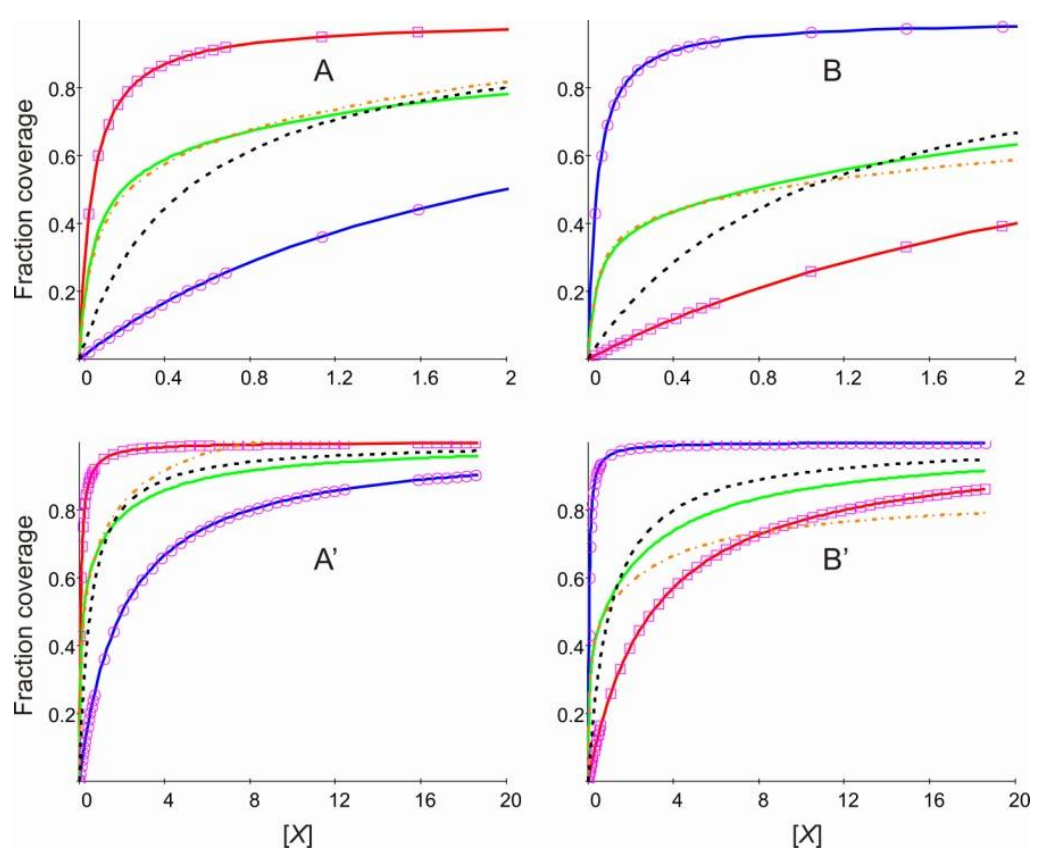

Figure 2. Isotherms expressed as a function of the concentration of $[X]$. Left: Calculated for $K_{1}=50.0$ and $K_{10}=1.0$. Right: Calculated for $K_{1}=1.0$ and $K_{10}=50.0 .(A, B)$ in the range of $[X]$ between 0 and 2, and $\left(\mathbf{A}^{\prime}, \mathbf{B}^{\prime}\right)$ between 0 and 20. Solid lines: Amount of the objects $X_{m}\{A B\} X_{n}$. Red: $m=1,2,3$, all $n$; divided by 3. Blue: $\{A B\} X_{n}, n=1,2$; divided by 2 . The rectangles and the circles correspond to Langmuir's eqs. (29) and (29A) of ref. [1] with $K_{L}=K_{1} / 3$ and $K_{L}=K_{10} / 2$, respectively. Green: all $X_{m}\{A B\} X_{n}$, except $\{A B\}$, divided by 5. Black, dashed: Isotherm calculated using Langmuir's eqs. (29) and (29A) of ref. [1] with optimized values for $K_{L}$. Orange, dash dot: Sum of the red and the blue curves weighted by an optimized factor.

It is interesting but not surprising that the amount of the objects $X_{m}\{A B\} X_{n}(m=1,2,3$, all $n$; divided by 3) can be perfectly described by Langmuir's isotherm equation. We observe the same for the concentration of $\{A B\} X_{n}(n=1,2$; divided by 2$)$. The sum of all objects $X_{m}\{A B\} X_{n}(m=0,1,2,3$, all $n)$, however, cannot be described by the Langmuir isotherm equation. This behaviour seems to be of general validity, as I have numerically tested for a number of representative examples. It should be possible to prove this analytically but such a proof is not yet known. If the numerical values of $K_{1}$ and $K_{10}$ are equal, the system simplifies to the situation we have discussed in ref. [1]. In the other extreme, when $K_{1}$ and $K_{10}$ differ by orders of magnitude, the system decomposes into separate parts.

Different types of explanations for isotherms that deviate from Langmuir isotherms have been developed. They are in many cases satisfactory because they have been linked to a microscopic phenomenon, but they seem to be arbitrary in other situation $[6,11,18,24]$. We find that it is sufficient to have two different sites in a multiple equilibrium in order to observe a characteristic that differs from Langmuir's equation, despite of the fact that the latter applies for individual parts. Writing multiple chemical equilibria could therefore be useful for explaining experimental data and for making prediction. Instead of inventing adsorption models, it might make sense to describe a system in such terms. The system may consist of one set of equivalent sites [1], two sets, as reported here, or even of several sets of equivalent sites [16].

Conflicts of Interest: The authors declare no conflict of interest.

\section{Appendix A}

We express the equilibrium equations of the reaction sin Table 1 by means of the stoichiometry matrix; see refs. $[9,10]$, where the labels with a bar are the logarithm of the corresponding object: $\overline{\text { value }}=\log ($ value $)$. This leads to Equation (A1). 


$$
\left[\begin{array}{ccccccccccccc}
1 & -1 & 0 & 0 & 0 & 0 & 0 & 0 & 0 & 0 & 0 & 0 & -1 \\
0 & 1 & -1 & 0 & 0 & 0 & 0 & 0 & 0 & 0 & 0 & 0 & -1 \\
0 & 0 & 1 & 0 & 0 & 0 & 0 & 0 & 0 & -1 & 0 & 0 & -1 \\
0 & 0 & 0 & 1 & -1 & 0 & 0 & 0 & 0 & 0 & 0 & 0 & -1 \\
0 & 0 & 0 & 0 & 1 & -1 & 0 & 0 & 0 & 0 & 0 & 0 & -1 \\
0 & 0 & 0 & 0 & 0 & 1 & 0 & 0 & 0 & 0 & -1 & 0 & -1 \\
0 & 0 & 0 & 0 & 0 & 0 & 1 & -1 & 0 & 0 & 0 & 0 & -1 \\
0 & 0 & 0 & 0 & 0 & 0 & 0 & 1 & -1 & 0 & 0 & 0 & -1 \\
0 & 0 & 0 & 0 & 0 & 0 & 0 & 0 & 1 & 0 & 0 & -1 & -1 \\
0 & 0 & 0 & 0 & 0 & 0 & 0 & 0 & 0 & 1 & -1 & 0 & -1 \\
0 & 0 & 0 & 0 & 0 & 0 & 0 & 0 & 0 & 0 & 1 & -1 & -1
\end{array}\right]\left(\begin{array}{c}
\frac{X_{3}\{A B\} X_{2}}{\overline{X_{2}\{A B\} X_{2}}} \overline{\overline{X\{A B\} X_{2}}} \\
\overline{X_{3}\{A B\} X} \\
\frac{X_{2}\{A B\} X}{\bar{X}\{A B\} X} \\
\frac{\overline{X_{3}\{A B\}}}{\bar{X}_{2}\{A B\}} \\
\frac{X\{A B\}}{\overline{\{A B\} X_{2}}} \\
\overline{\{A B\} X} \\
\overline{\{A B\}} \\
\bar{X}
\end{array}\right)=\left(\begin{array}{c}
\overline{K_{3}} \\
\overline{K_{1}} \\
\overline{K_{6}} \\
\overline{K_{5}} \\
\overline{K_{9}} \\
\overline{K_{8}} \\
\overline{K_{7}} \\
\overline{K_{10}}
\end{array}\right)
$$

Linear transformation of (A1) allows finding the solution (A2); see e.g., ref. [10].

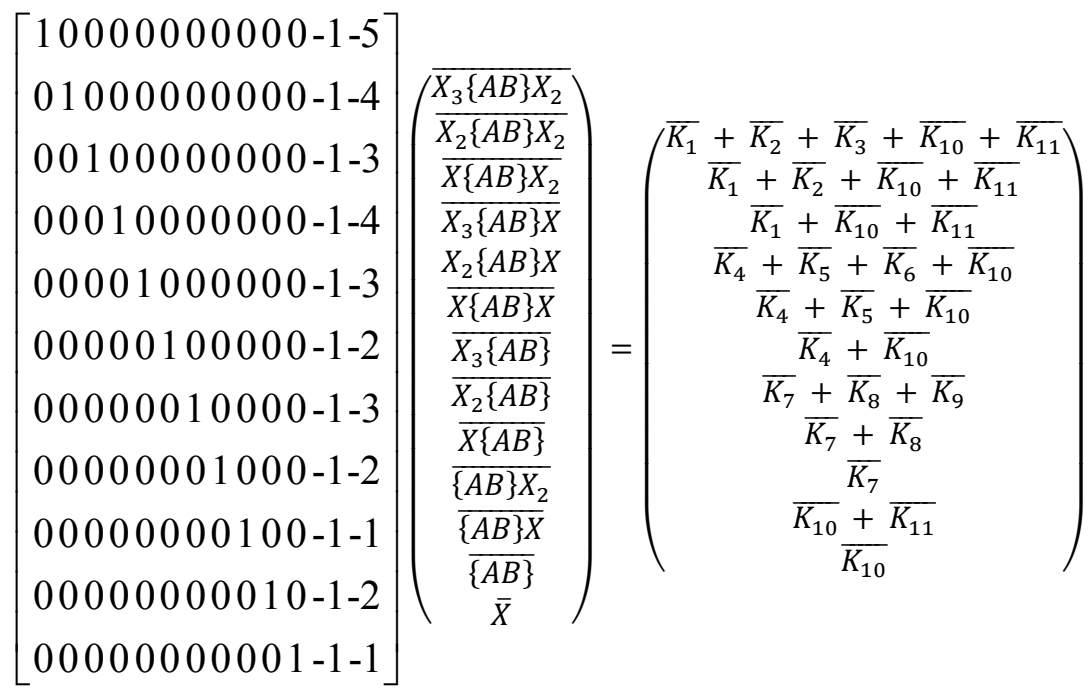

Using this solution we can express the concentrations of the $X_{m}\{A B\} X_{n}$ species as reported in Table A1. $[X]$ is considered to be a parameter, while the concentration $C_{12}=\{A B\}$ can be determined for the condition that in a closed system the total concentration of the $X_{m}\{A B\} X_{n}$ species, which we name $A_{0}$, is constant, a fact which we express in Equation (A3) as follows:

$$
A_{0}-\sum_{i=1}^{12} C_{i}=0
$$

\begin{tabular}{|c|c|c|}
\hline$C_{1}=$ & {$\left[X_{3}\{A B\} X_{2}\right]$} & $=[\{A B\}][X]^{5} K_{1} K_{2} K_{3} K_{10} K_{11}$ \\
\hline $\mathrm{C}_{2}=$ & {$\left[X_{2}\{A B\} X_{2}\right]$} & $=[\{A B\}][X]^{4} K_{1} K_{2} K_{10} K_{11}$ \\
\hline$C_{3}=$ & {$\left[X\{A B\} X_{2}\right]$} & $=[\{A B\}][X]^{3} K_{1} K_{10} K_{11}$ \\
\hline $\mathrm{C}_{4}=$ & {$\left[X_{3}\{A B\} X\right]$} & $=[\{A B\}][X]^{4} K_{4} K_{5} K_{6} K_{10}$ \\
\hline $\mathrm{C}_{5}=$ & {$\left[X_{2}\{A B\} X\right]$} & $=[\{A B\}][X]^{3} K_{4} K_{5} K_{10}$ \\
\hline $\mathrm{C}_{6}=$ & {$[X\{A B\} X]$} & $=[\{A B\}][X]^{2} K_{4} K_{10}$ \\
\hline$C_{7}=$ & {$\left[X_{3}\{A B\}\right]$} & $=[\{A B\}][X]^{3} K_{7} K_{8} K_{9}$ \\
\hline$C_{8}=$ & {$\left[X_{2}\{A B\}\right]$} & $=[\{A B\}][X]^{2} K_{7} K_{8}$ \\
\hline $\mathrm{C}_{9}=$ & {$[X\{A B\}]$} & $=[\{A B\}][X] K_{7}$ \\
\hline$C_{10}=$ & {$\left[\{A B\} X_{2}\right]$} & $=[\{A B\}][X]^{2} K_{10} K_{11}$ \\
\hline$C_{11}=$ & {$[\{A B\} X]$} & $=[\{A B\}][X] K_{10}$ \\
\hline
\end{tabular}

Table A1. Concentrations $C_{i}$, calculated using Equation (A2). 
Figures $\mathrm{A} 1$ and $\mathrm{A} 2$ show an example calculate with different equilibrium constants, namely: $\left(\mathrm{A}, \mathrm{A}^{\prime}\right) K_{1}=5$ and $K_{10}=1 ;\left(\mathrm{B}, \mathrm{B}^{\prime}\right) K_{1}=1$ and $K_{10}=5$. We observe a different behavior with respect to Figures 1 and 2, as expected.
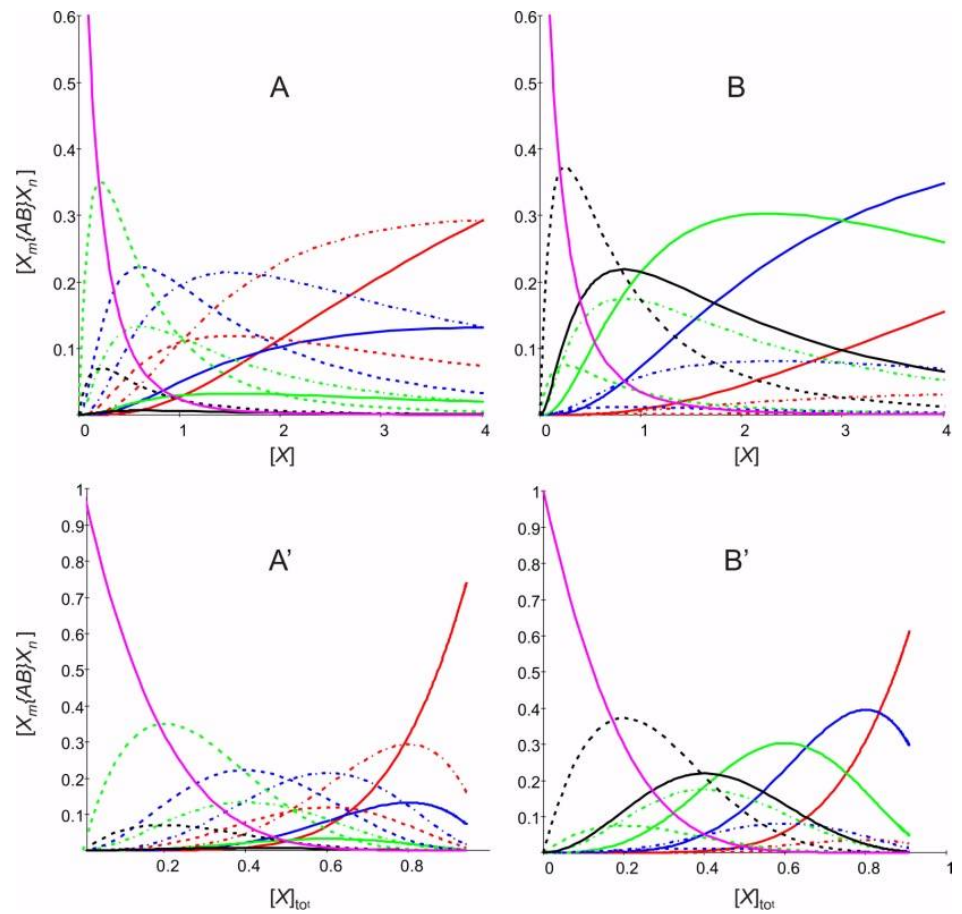

Figure A1. Concentration of $X_{m}\{A B\} X_{n}$ as a function of the concentration of $X,(\mathbf{A}, \mathbf{B})$, and versus the total concentration $[X]_{\text {tot }}$ of $X$ in the system, $\left(\mathbf{A}^{\prime}, \mathbf{B}^{\prime}\right)$. $\left(\mathbf{A}, \mathbf{A}^{\prime}\right)$ calculated for $K_{1}=5.0$ and $K_{10}=1.0 ;\left(\mathbf{B}, \mathbf{B}^{\prime}\right)$ calculated for $K_{1}=1.0$ and $K_{10}=5.0$. Red lines: $\left[X_{3}\{A B\} X_{n}\right]$ (solid: $n=2$, dash: $n=1$, dash dot: $n=0$ ). Blue lines: $\left[X_{2}\{A B\} X_{n}\right]$ (solid: $n=2$, dash: $n=1$, dash dot: $n=0$ ). Green lines: $\left[X\{A B\} X_{n}\right]$ (solid: $n=2$, dash: $n=1$, dash dot: $n=0)$. Black lines: $\left[\{A B\} X_{2}\right]$ solid: $[\{A B\} X]$ dash. Pink line: $[\{A B\}]=C_{12}$ (solid).
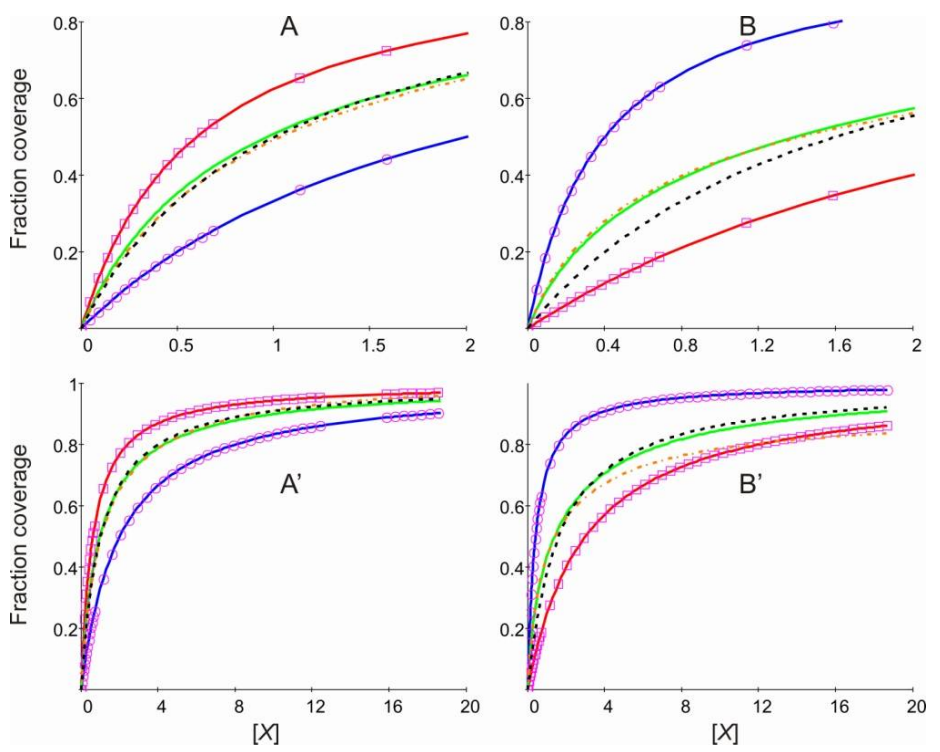

Figure A2. Isotherms expressed as a function of the concentration of $[X]$. $\left(\mathbf{A}^{\prime}\right)$ calculated for $K_{1}=5.0$ and $K_{10}=1.0 ;\left(\mathbf{B}^{\prime}\right)$ calculated for $K_{1}=1.0$ and $K_{10}=5.0 .(\mathbf{A}, \mathbf{B})$ in the range of $X$ between 0 and 2 and $\left(\mathbf{A}^{\prime}, \mathbf{B}^{\prime}\right)$ between 0 to 6 . Solid lines: Amount of the objects $X_{m}\{A B\} X_{n}$. Red: $m=1,2,3$, all $n$; divided by 3 . Blue: $\{A B\} X_{n}, n=1,2$; divided by 2 . The rectangles and the circles correspond to Langmuir's eqs. (29) and (29A) of ref. [1] with $K_{L}=K_{1} / 3$ and $K_{L}=K_{10} / 2$, respectively. Green: all $X_{m}\{A B\} X_{n}$, except $\{A B\}$, divided by 5. Black, dashed: Isotherm calculated using Langmuir's eqs. (29) and (29A) of ref. [1] with optimized values for $K_{L}$. Orange, dash dot: Sum of the red and the blue curves weighted by an optimized factor. 


\section{References}

1. Calzaferri, G. Entropy in multiple equilibria, theory and applications. Phys. Chem. Chem. Phys. 2017, 19, 10611-10621.

2. Langmuir, I. The constitution and fundamental properties of solids and liquids. Part I. Solids. J. Am. Chem. Soc. 1916, 38, 2221-2295.

3. Langmuir, I. The adsorption of gases on plane surfaces of glass, mica and platinum. J. Am. Chem. Soc. 1918, 40, 1361-1403.

4. Denbigh, K. The Principles of Chemical Equilibrium, 2rd ed.; Cambridge University Press: Cambridge, UK, 1966.

5. Tabacchi, G. Supramolecular organization and confined nanospaces. Chem. Phys. Chem. 2018, in press.

6. Barrer, R.M. Zeolites and Clay Minerals as Sorbents and Molecular Sieves; Academic: London, UK, 1978; ISBN 0-12-079350-4.

7. Nagaj, J.; Stokowa-Sołtys, K.; Kurowska, E.; Frączyk, T.; Jeziowska-Bojczuk, M.; Bal, W. Revised coordination model and stability constants of Cu (II) complexes of tris buffer. Inorg. Chem. 2013, 52, 1392713933.

8. David, C.; Companys, E.; Galceran, J.; Garcés, J.L.; Mas, F.; Rey-Castro, C.; Salvador, J.; Puy, J. Competitive $\mathrm{Cd} 2+/ \mathrm{H}+$ complexation to polyacrylic acid described by the stepwise and intrinsic stability constants. J. Phys. Chem. 2008, 112, 10092-10100.

9. Prigogine, I. Thermodynamics of Irreversible Processes, 3rd ed.; Wiley \& Sons: Hoboken, NJ, USA, 1967.

10. Dubler, T.; Maissen, C.; Calzaferri, G. Diskussion Chemischer Gleichgewichte. Z. Naturforsch. 1976, 31, 569579.

11. Breck, D.W. Zeolite Molecular Sieves; John Wiley: New York, NY, USA, 1974; ISBN 0-471-09985-6.

12. Schoonheydt, R.A.; Weckhuysen, B.M. Editorial Highlight: Molecules in confined spaces. Phys. Chem. Chem. Phys. 2009, 11, 2794-2798.

13. Schulz-Ekloff, G.; Wöhrle, D.; van Duffel, B.; Schoonheydt, R.A. Chromophores in porous silicas and minerals: Preparation and optical properties. Microporous Mesoporous Mater. 2002, 51, 91-138.

14. Craciun, G.; Feinberg, M. Multiple Equilibria in Complex Chemical Reaction Networks: Semiopen Mass Action Systems. SIAM J. Appl. Math. 2010, 70, 1859-1877.

15. Roduner, E.; Radhakrishnan, S.G. In command of non-equilibrium. Chem. Soc. Rev. 2016, 45, $2768-2784$.

16. Meyer, M.; Leiggener, C.; Calzaferri, G. Particle distribution in a microporous material: Experiments with zeolite A. Chem. Phys. Chem. 2005, 6, 1071-1080.

17. Calzaferri, G.; Huber, S.; Maas, H.; Minkowski, C. Host-guest antenna materials. Angew. Chem. Int. Ed. 2003, 42, 3732-3758.

18. Valtchev, V.; Mintova, S.; Tsapatsis, M. (Eds.) Ordered Porous Solids, 1st ed.; Elsevier: Oxford, UK, 2009; ISBN 978-0-444-53189-6.

19. Sola-Llano, R.; Martínez-Martínez, V.; Fujita, Y.; Hortigüela, L.G.; Alfayate, A.; Uji-I, H.; Fron, E.; PérezPariente, J.; López-Arbeloa, I. Formation of a nonlinear optical host-guest hybrid material by tight confinement of LDS 722 into aluminophosphate 1D nanochannels. Chem. Eur. J. 2016, 22, 15700-15711.

20. Al-Aqar, R.; Atahan, A.; Benniston, A.C.; Perks, T.; Waddell, P.G.; Harriman, A. Exciton Migration and Surface Trapping for a Photonic Crystal Displaying Charge-Recombination Fluorescence. Chem. Eur. J. 2016, 22, 15420-15429.

21. Ramamurthy, V.; Inoue, Y. (Eds.) Supramolecular Photochemistry: Controlling Photochemical Processes; John Wiley \& Sons: Hoboken, NJ, USA, 2011; ISBN 978-0-470-23053-4.

22. Tabacchi, G.; Fois, E.; Calzaferri, G. Structure of Nanochannel Entrances in Stopcock-Functionalized Zeolite L Composites. Angew. Chem. Int. Ed. 2015, 54, 11112-11116.

23. Tabacchi, G.; Calzaferri, G.; Fois, E. One-dimensional self-assembly of perylene-diimide dyes by unidirectional transit of zeolite channel openings. Chem. Commun. 2016, 52, 11195-11198.

24. Cejka, J.; van Bekkum, H.; Corma, A.; Schüth, F. (Eds.) Studies in Surface Science and Catalysis, Vol. 168: Introduction to Zeolite Science and Practice, 3rd ed.; Elsevier: Amsterdam, The Netherlands, 2007; ISBN 9780-444-53063-9.

25. Gigli, L.; Arletti, R.; Vitillo, J.G.; Alberto, G.; Martra, G.; Devaux, A.; Vezzalini, G. Thionine dye confined in zeolite L: synthesis location and optical properties. J. Phys. Chem. C 2015, 119, 16156-16165.

26. Getman, R.B.; Bae, J.S.; Wilmer, C.E.; Snurr, R.Q. Review and analysis of molecular simulations of methane, hydrogen, and acetylene storage in metal-organic frameworks. Chem. Rev. 2012, 112, 703-723. 
27. Lencione, D.; Gehlen, M.H.; Trujillo, L.N.; Leitao, R.C.F.; Albuquerque, R.Q. The spatial distribution of the photostability of thionine in zeolite L nanochannels investigated by Photobleaching Lifetime Imaging Microscopy. Photochem. Photobiol. Sci. 2016, 15, 398-404.

28. Bussemer, B.; Dreiling, I.; Grummt, U.-W.; Mohr, G.J. Spectroscopic and quantum chemical study of the Brønsted acid sites in zeolite L channels with acidochromic cyanine dyes. J. Photochem. Photobiol. A Chem. 2009, 204, 90-96.

29. Woodtli, P.; Giger, S.; Müller, P.; Sägesser, L.; Zucchetto, N.; Reber, M.J.; Ecker, A.; Brühwiler, D. Indigo in the nanochannels of zeolite L: Towards a new type of colorant. Dyes Pigment. 2017, doi:10.1016/j.dyepig.2017.10.029.

30. Kunzmann, A.; Seifert, R.; Calzaferri, G. Particle distribution in a microporous material. J. Phys. Chem B 1999, 103, 18-26.

31. Van Speybroeck, V.; Hemelsoet, K.; Joos, L.; Waroquier, M.; Bell, R.G.; Catlow, C.R.A. Advances in theory and their application within the field of zeolite chemistry. Chem. Soc. Rev. 2015, 44, 7044-7111.

32. Gigli1, L.; Arletti, R.; Tabacchi, G.; Fabbiani, M.; Vitillo, J.G.; Gianmario, M.; Devaux, A.; Miletto, I.; Quartieri, S.; Calzaferri, G.. Structure and Host-Guest Interactions of Perylene-Bisimide Dyes in Zeolite L Nanochannels. J. Phys. Chem. C 2018, in press.

(C) 2018 by the author. Licensee MDPI, Basel, Switzerland. This article is an open access article distributed under the terms and conditions of the Creative Commons Attribution (CC BY) license (http://creativecommons.org/licenses/by/4.0/). 